\title{
Effects of cold tandem rolling on microstructures and mechanical properties of TC16 titanium alloy wire for fastener use
}

SUN Xiaoping, HOU Fengqi, YANG Hui, Wang Kaixuan, LUU Xianghong

$$
\text { Xi'an, 710018, China }
$$

Abstract : High-precision dimension controlling and special requirement of microstructures and mechanical for TC16 Alloy wire was obtained by cold rolling. In this paper, the effects of cold tandem rolling on M and STA mechanical of TC16 Alloy wire were investigated through Comparative study TC16 Alloy wire by cold tandem rolling and hot-draw. The results show that the M state tensile and yield strength of TC16 Alloy wire obtained by cold tandem was lower, while the ductility was higher. And this benefits the subsequent Cold Heading formability. The result of solid solution for TC16 Alloy wire by cold tandem rolling was evidenced, the tensile strength has been increased and the ductility has been reduced more drastically. To break down elongated a of Vertical microstructure ability and drastic machining deformation have significant contributions to the annealed ductility and solution strengthening. It is very positive to TC16 titanium alloy use for fastener of heat treatment strength with the cold tandem rolling microstructure ability

\section{Introduction}

Cold rolled wire not only has good surface quality and high dimensional accuracy, but also has better microstructure and properties. The reasonable coordination of cold rolling deformation and heat treatment can not only easily meet the requirements of users for processing quality and comprehensive performance, but also be particularly beneficial to the production of some important products requiring special structure and performance $e^{[1]}$

TC16 titanium alloy containing a small amount of Al, the more stable $\beta$ elements $(\mathrm{Mo}, \mathrm{V}), \beta$ stability coefficient $\mathrm{K}_{\beta}$ is as high as 0.8 , which has high specific strength, good plasticity, the least sensitivity to notch stress concentration and torsional stress concentration at solid solution aging condition. It is a typical solid solution aging fortified $a+\beta$ titanium alloy, be widely used in aviation, aerospace fasteners and parts ${ }^{[2-4]}$. $T C 16$ titanium alloy has good deformation ability at room temperature and can be directly formed by cold upsetting after annealing. Cold upsetting forming is adopted for fasteners, which has the advantages of high machining precision, good surface quality, high production efficiency, easy operation conditions and low processing cost. It is the preferred technological direction for the engineering production of TC16 titanium alloy fasteners in China. However, the annealed microstructure and properties of TC16 alloy wires must be strictly controlled to realize cold upsetting processing stably, and the properties of the fasteners after cold upsetting forming must be improved obviously after solution aging strengthening, so as to realize the processing and application of heat treatment strengthening cold upsetting TC16 titanium alloy fasteners ${ }^{[5-7]}$.

The microstructure and properties of TC16 titanium alloy formed by cold upsetting were comprehensively analyzed by domestic scholars and research institutions through heat treatment process, phase structure and deformation-induced martensite ${ }^{[8-9]}$. Based on the Johnson-cook constitutive model, Yangyang ${ }^{[10]}$ et al. obtained the parameters of the constitutive model of TC16 alloy by fitting experimental data, which provided a reference for the cold upsetting deformation of TC16 alloy. Zhang zhengang ${ }^{[11]}$ et al. described the quantitative changes of microstructure of TC16 titanium alloy under different heat treatment processes, and the reasonable heat treatment process can significantly improve the strengthening effect of TC16 alloy for cold upsetting. However, due to
the limitation of equipment condition, the current domestic processing and preparation of TC16 titanium alloy wire limited to hot rolling, hot drawing or both the processing way of combining. Eventually, the properties of TC16 alloy wire which was obtained cannot meet the stability requirements of cold upsetting for TC16 fasteners, while to adopt the new cold rolling process of the preparation of cold upsetting TC16 titanium alloy wire is used to study less.

Therefore, in this paper, the effects of the cold tandem rolling process on the microstructure and properties of TC16 titanium alloy wires for cold heading were studied by comparing the annealing and solution aging properties of the TC16 titanium alloy wires prepared by the cold tandem rolling method and the traditional hot drawing method.

\section{$\underline{\text { 2. Experiment }}$}

The experimental materials used in this study are $\$ 9.5 \mathrm{~mm}$ coiled wire of $\mathrm{TCl} 16$ (Ti-3Al-5Mo-4.5V) titanium alloy which were processed by western superconducting technology co., Ltd., Its chemical composition (wto)is: $2.5 \sim 3.8 \mathrm{Al}, 4.5 \sim 5.5 \mathrm{Mo}, 4.0 \sim 5.0 \mathrm{~V}$, $0.10 \sim 0.30 \mathrm{Si}, 0.05 \sim 0.15 \mathrm{O}$ allowance for Titanium. Preparation process for $\Phi 9.5 \mathrm{~mm} \mathrm{TC} 16$ titanium alloy were ingot to breakdown, forging, precision forging and rolling. This $\Phi 9.5 \mathrm{~mm}$ bar was peeled to $\Phi 8.5 \mathrm{~mm}$ after annealing, and then it was used in cold tanden
rolling and hot drawing.

Cold tandem rolling is a kind of processing method which adopts imported 90D cold tandem rolling machine. Its working principle is to obtain special-shaped wire with 12 -side shape in cross section through 10 pass rolling of cold tandem rolling, and then tha special-shaped wire was rolled to $\$ 4.0 \mathrm{~mm}$ wire by adding two cold-rolling gauge circle units of the frame equivalently to be finishing rolling. Hot drawing processing is
obtained from $\$ 4.0 \mathrm{~mm}$ wire which were prepared in the above two ways respectively. Table 1 shows the heat-treatment for the sample with the two processing methods.

Table 1 Technology for heating processing for Dia. $4.0 \mathrm{~mm}$ TC16 alloy with the two Processing method

\begin{tabular}{|c|c|c|}
\hline Processing method & Heat-treated states & Heat-treatment \\
\hline \multirow{2}{*}{ Cold tandem rolling } & $\mathrm{M}$ & $780^{\circ} \mathrm{C} / 2 \mathrm{~h}, \mathrm{FC}$ 至 $550^{\circ} \mathrm{C}, \mathrm{AC}$ \\
\cline { 2 - 3 } & STA & $800^{\circ} \mathrm{C} / 2 \mathrm{~h}, \mathrm{WQ}+560^{\circ} \mathrm{C} / 8 \mathrm{~h}, \mathrm{AC}$ \\
\hline \multirow{2}{*}{ Hot-draw } & $\mathrm{M}$ & $780^{\circ} \mathrm{C} / 2 \mathrm{~h}, \mathrm{FC}$ 至 $550^{\circ} \mathrm{C}, \mathrm{AC}$ \\
\cline { 2 - 3 } & STA & $800^{\circ} \mathrm{C} / 2 \mathrm{~h}, \mathrm{WQ}+560^{\circ} \mathrm{C} / 8 \mathrm{~h}, \mathrm{AC}$ \\
\hline
\end{tabular}

\section{Results and Discussion}

3.1 Comparison of annealed microstructure and properties

Transverse and Longitudinal microstructure of sample of $\Phi 8.5 \mathrm{~mm}$ billet which were heated treating for 2 hour at $780^{\circ} \mathrm{C}$ then cooling with furnace temperature to $550^{\circ} \mathrm{C}$ were observed. The transverse microstructure of $\Phi 8.5 \mathrm{~mm}$ billet is small near-baske Transverse and Longitudinal microstructure of sample of $\varnothing 8.5 \mathrm{~mm}$ billet which were heated treating for 2 hour at $780^{\circ} \mathrm{C}$
microstructure, and the longitudinal microstructure is elongated microstructure in the rolling direction, as shown in FIG.

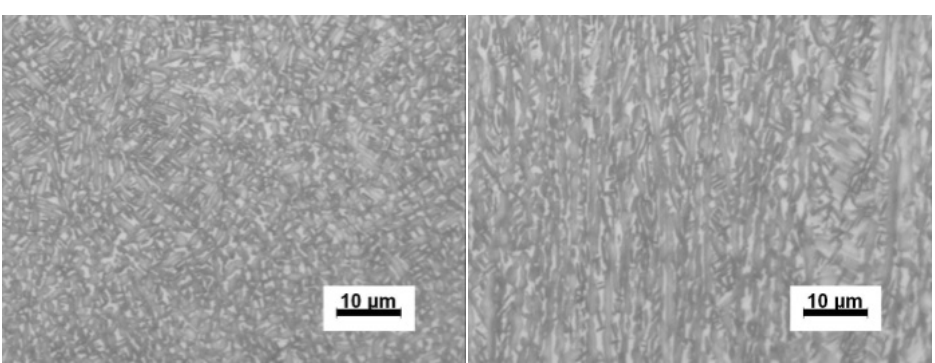

(a) transverse microstructure, 1000x (b) longitudinal microstructure, 1000x

Fig.1 Microstructures of Dia. 8.5mm TC16 alloy annealed wire

This $\Phi 4.0 \mathrm{~mm}$ wire was obtained by $\Phi 8.5 \mathrm{~mm}$ billet through 8 pass drawing. FIG.2 shows the transverse and longitudinal microstructure of the $\Phi 4.0 \mathrm{~mm}$ wire sample after heated for 2 hour at $780^{\circ} \mathrm{C}$ then cooling with furnace temperature to $550^{\circ} \mathrm{C}$. Althoug dynamic recovery exists in the process of hot drawing at the two-phase dynamic recovery exists in the process of hot drawing at the two-phase temperature, the drawing deformation is a gradual transition from the edge to the center of the wire, and the amount of inter-pass deformation is small, so the original longitudinal elongation of
phase structure of the wire billet cannot be broken. At the same time, during the drawing process, part of phase will be elongated along the drawing direction, resulting in obvious fibrous tissue, as shown in FIG. 2 (b). Therefore, the texture of longitudinal rolling
after hot drawing at two-phase temperature is elongated to a structure in which both alpha phase and equiaxed alpha coexist.

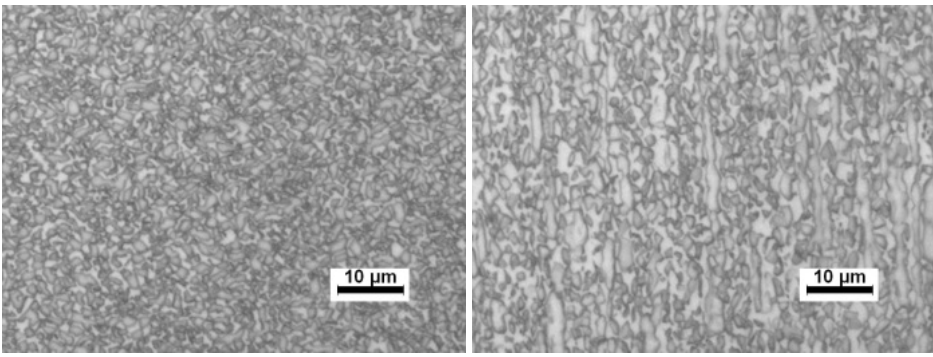

(a) transverse, 1000x; (b) longitudinal, 1000x

Fig.2 Microstructures for Dia. $4.0 \mathrm{~mm}$ TC16 alloy as annealed by Hot-draw

This $\Phi 4.0 \mathrm{~mm}$ wire was obtained by $\Phi 8.5 \mathrm{~mm}$ billet through 10 pass rolling and 2 pass finishing rolling. FIG.3 shows the transverse and longitudinal microstructure of the $\Phi 4.0 \mathrm{~mm}$ wire sample after heated for 2 hour at $780^{\circ} \mathrm{C}$ thencooling with furnace temperature to to $550^{\circ} \mathrm{C}$. Compared with hot drawing, cold tandem rolling has more severe deformation. The original longitudinal elongation of phase microstructure can be effectively broken at room temperature, and the obtained longitudinal microstructure after

(C) The Authors, published by EDP Sciences. This is an open access article distributed under the terms of the Creative Commons Attribution License 4.0 (http://creativecommons.org/licenses/by/4.0/). 


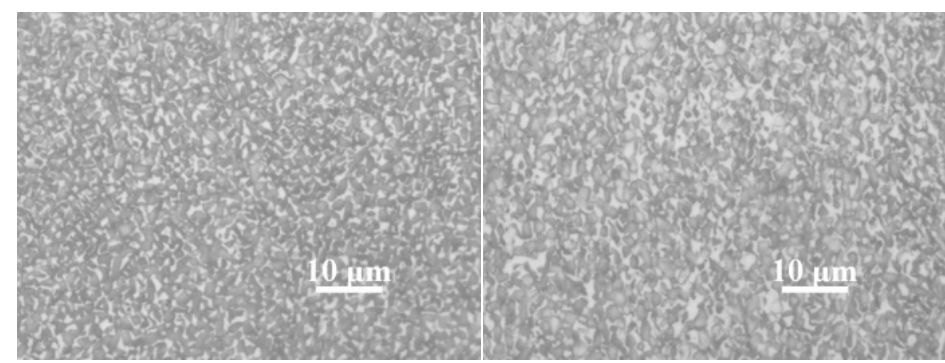

(a) transverse, 1000x ; (b) longitudinal, 1000x
Fig. 3 Microstructures for Dia. $4.0 \mathrm{~mm}$ TC16 alloy as annealed by Cold tandem rolling

After annealing, the tensile properties of the wires with different structure states were tested according to GB/T 228 at room temperature. The test results of tensile strength (Rm), yield strength (Rp0.2) and elongation (A) were shown in FIG.4.
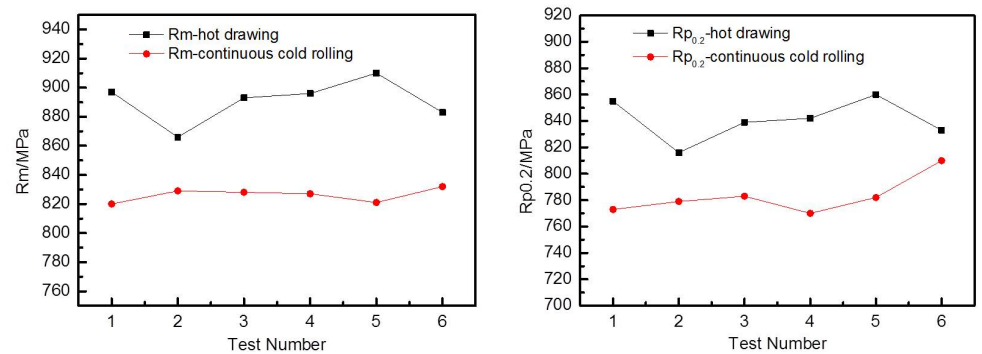

(a) The tensile strength; (b) The yield strength

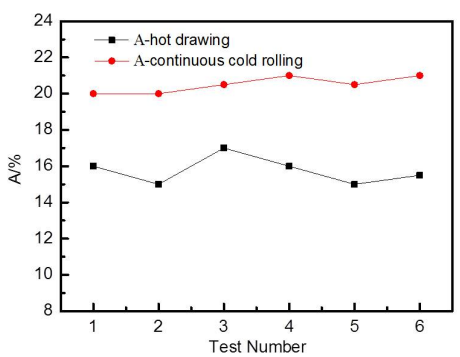

(c) The ductility
Fig. 4 Tension test of Dia. $4.0 \mathrm{~mm}$ TC16 alloy with the two Processing method

The tensile properties of TC16 titanium alloy $\$ 4.0 \mathrm{~mm}$ wire, which were processed by cold tandem rolling and hot drawing methods respectively after same annealing were analyzed. The contrast shows that TC16 titanium alloy wires tensile strength and yield strength of cold tandem rolling are lower than that of hot drawing, but the plasticity is better than that of hot drawing.

3.2 Cold heading forming properties of wire in two processing methods

The TC16 titanium alloy $\$ 4.0 \mathrm{~mm}$ wire, which were processed by cold tandem rolling and hot drawing methods respectively after same annealing were carried out in accordance with the HB 8015-2002(Hexagon head bolts in titanium alloy TC16 with tolerance f9) to cold heading $44 \mathrm{~mm} \times 20 \mathrm{~mm}$ fasteners, eventually cold heading fasteners, as shown in FIG 5 .
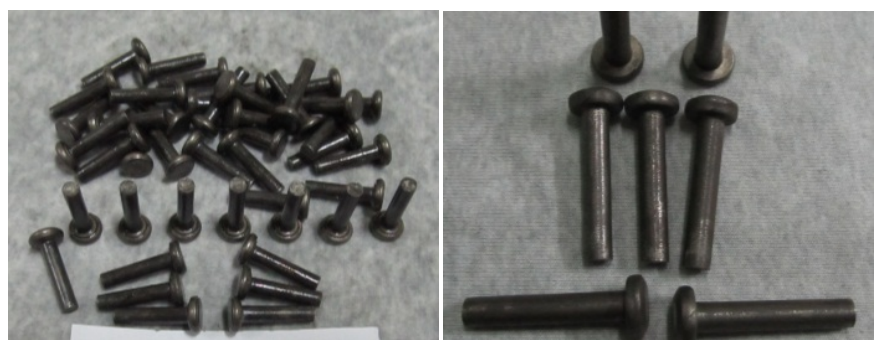
(a) Fastener samples of Dia.4mm wire by Cold tandem rolling; (b) Fastener samples of Dia.4mm wire by Hot-draw
Fig. 5 Cold -upseting fastener samples of TC16 alloy with the two Processing method

TC16 titanium alloy such as Dia.4mm wires with equal axial a phase in transverse and longitudinal direction obtained by cold continuous rolling have low strength and high plasticity. That optimum process performance makes the fastener deform well in the process of cold heading. Thus, the surface of the cold heading fastener is free from cracks, strains and other defects, as shown in FIG. 5 (a). While, the horizontal direction is equiaxed a phase and the longitudinal direction is strip a phase of the TC16 titanium alloy Dia.4mm wires which was obtained by hot drawing that have low strength and high plasticity. That Poor performance prevents the fasteners from deforming well during the cold heading forming process. Thus, the surface of the cold heading fastener is cracked and the rod is scratched, as shown in FIG. 5 (b).

The cold heading process of fasteners is longitudinal microstructure of the material ${ }^{[12-14]}$.

The longitudinal elongated microstructure is less compatible with the longitudinal basket microstructure and the longitudinal isoaxial microstructure during cold heading deformation. Compared with the longitudinal basket and the longitudinal equiaxed microstructure, the lengthwise elongated microstructure is less changeable in the process of cold heading deformation, Therefore, for TC16 titanium alloy wire used in cold heading, the longitudinal wire with elongated microstructure is more likely to crack during cold heading forming, while the fine equiaxed microstructure and net basket microstructure are conducive to cold heading forming ${ }^{[15,16]}$ 

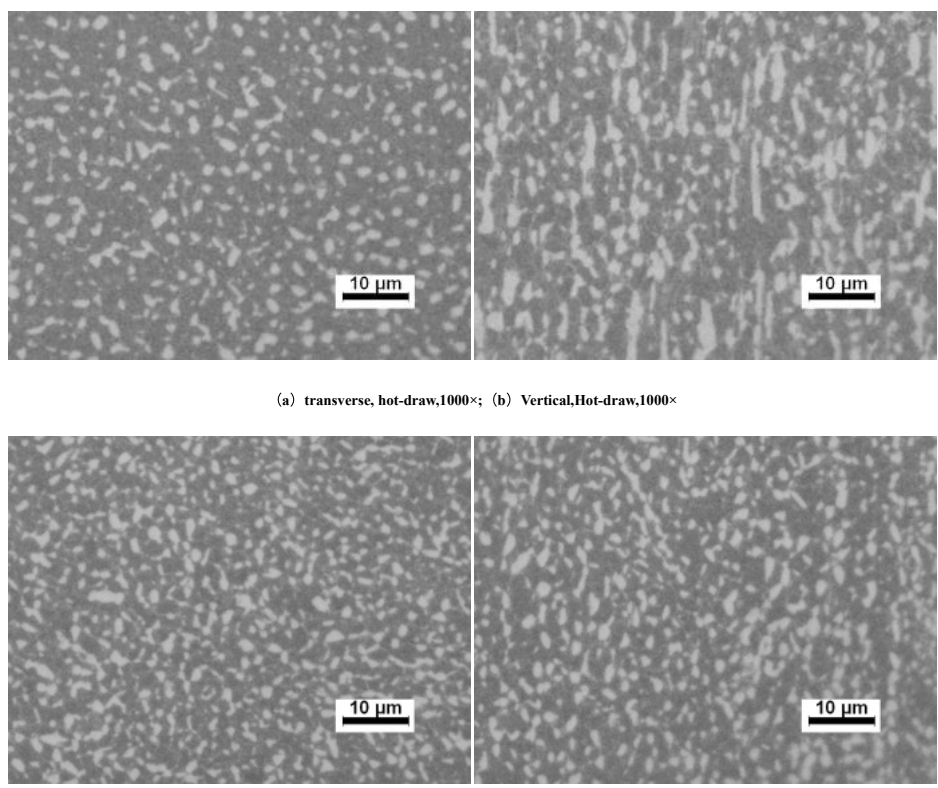

(c) horizontal, continuous cold rolling,1000×; (d) Vertical, continuous cold rolling,1000× Fig.6 Microstructures for Dia. 4.0mm TC16 alloy of STA state with the two Processing method

After the solution aging, the transverse and longitudinal microstructure of hot-drawn wire retained the annealed microstructure, and the transverse microstructure was composed of isoaxial a phase, and the longitudinal microstructure was composed of elongated a phase and isoaxial a phase. But the overall phase content was less than that of cold tandem rolling. The transverse and longitudinal microstructure of cold continuous rolling wire after solution aging were all composed of equiaxed a phase.

The tensile properties of the two processing methods were tested, and the test comparison results were shown in FIG.7
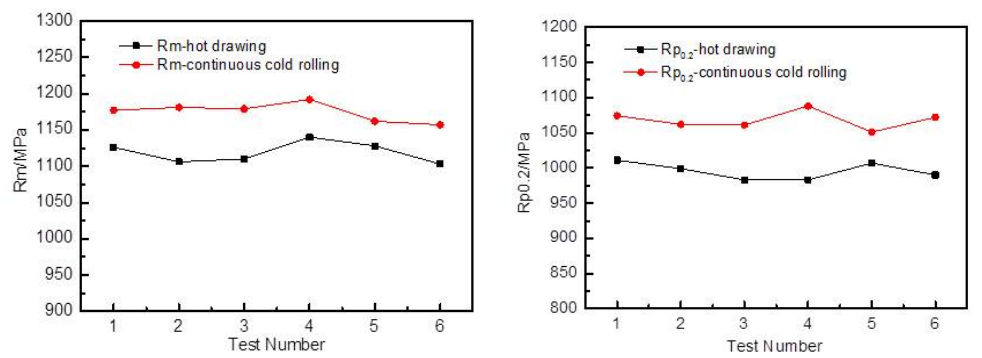

(a) The tensile strength Comparative ; (b) The yield strength Comparative
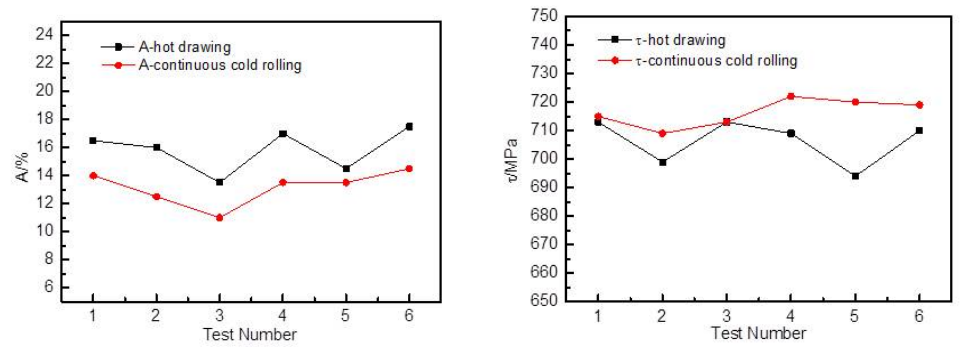

(c) The ductility Comparative ; (d) The shear strength Comparative

(c) The ductility Comparative; (d) The shear strength Comparative
Fig.7 Tension and shear test of Dia. $4.0 \mathrm{~mm}$ TC16 alloy with the two Processing method

After the solution aging treatment, the tensile strength and yield strength of TC16 wires processed by hot drawing and cold tandem rolling were significantly improved, and the plasticity was decreased. However, the strengthening effect of TC16 wire processed by cold tandem rolling is more obvious after the solid solution aging treatment, especially the tensile strength, yield strength and shear strength of the wire are higher than that of hot drawing processing.

Compared with hot drawing process, TC16 alloy wire produced by cold tandem rolling process has lower tensile and yield strength after common annealing, and its superior plasticity provides a good basis for cold heading process of TC16 alloy fasteners. TC16 alloy wire produced by cold tandem rolling process has high tensile and yield strength and high shear properties after solid solution aging, which can improve the performance of fasteners after cold heading processing

\section{4. conclusion}

(1) The longitudinal structure of TC16 titanium alloy $\$ 4.0 \mathrm{~mm}$ wire processed by hot drawing is a mixed microstructure of equiaxed and strip phase. Cold tandem rolling process can effectively break the original longitudinal structure and obtain the alloy wire with equal axis $a$ phase in both transverse and longitudinal direction.

(2) The TC16 titanium alloy wire produced by cold tandem rolling process has lower strength and higher plasticity than that produced by hot drawing process. It deforms well in the process of cold heading. The surface of the cold heading fasteners is free from cracks, surface tear and other defects.

(3) After solid solution aging treatment, the strengthening effect of TC16 titanium alloy wire processed by cold tandem rolling is more effective than that of hot-drawn wire, which is manifested as higher tensile strength, vield strength and shear strength. The processing method of cold tandem rolling has positive significance for the TC16 titanium alloy which needs to be strengthened by heat treatment for the finished fasteners.

\section{Reference}

[1] Qi Kemin, Ding Hua. Material forming technology. beijing: Metallurgy industry press, 2006:453.

[2] Liu Quanming, Zhang Zhaohui, Liu Shifeng, Yang Hairying, Zhao Fucai, Lu Huihui. Research and Development of Aerospace Fasteners Made with TC16 Alloy. Hot working technology, 2014, 43 (04):1

[3] Zhao Qingyun, Xu Feng.Research progress of titanium alloy for aerospace fasteners. The Chinese Journal of Nonferrous Metals, 2010,20(1):1021-1023.

[4] Zhang Qingling, Wang Qingru, Li Xingwu. Material Selection Analysis for Titanium Alloy Fasteners in Aviation Industry. Material Engineering, 2007(1):12-15.

[5] Yang Yang, Dong Li-min, Guan Shaoxuan. Effect of Cold and hot upset on microstructures and properties of TC16 titanium alloy. The Chinese Journal of Nonferrous Metals,2010,20(01) :23-25.

[6] Li Yinghao, He Fei, Hou Fengqi. Effect of Treatment on Microstructure and Mechanical Properties of TC16 Titanium Alloy Bars. Titanium industry Progress,2014,31(04):20-23.

[7] Liu Fenglei. Development of Aeronautical Ti Alloy Fastener in China. Aeronautical Manufacturing Technology,2000(6) :39-41.

[8] Luo Yuanyuan, Zhao Yongqing, Xi Zhengping. Effect of Heat Treatment Process on Microstructure and Properties of Ti Alloy for Fasteners. Hot Working Technology,2009, 38 (18): 118-121. 
[9] L.X.Li, Y.Lou, L.B.Yang, D.S.Peng, K.P.Rao. Flow stress behavior and deformation characteristics of Ti-3Al-5V-5Mo compressed at elevated tempertures. Materials \& Design,2002(23) :451-457. [10] Yang Yang, Zeng Yi, Wang Bingfeng. Johnson-Cook dynamic constitutive relationship for TC16 titanium alloy. Transactions of Nonferrous Metals Society of China, 2007,17:466-470.

[11] Zhang Zhengang, Song Hao, Wu Guoqing, Huang Xu, Huang Zheng, Ruan Zhongjian. Quantitative Relation of Heat Treatment and Microstructure Characteristics of Ti-3Al-5Mo-5V. Material Engine

[12] Wang Fuqiang, Sha Chunpeng, Sun Xiaolan. The Cold upset microstructure and properties of TC16 titanium alloy. Journal of Materials and Metallurgy, 2013, 12 (03): $55-58$.

[13] Wu ChongZhou. Effects of Annealing Scheme on the Structure and Properties of TC16 Titanium Alloy. ACTA Metallurgical SINICA,2002(9):94-96.

[14] Wu ChongZhou, Li Xingwu. Study of deformation Characters of TC16 Titanium Alloys under Room Temperature. Titanium industry Progress, 2006,23(06) : 17-19.

15] Lai YunJin. Study on the formation mechanism of titanium alloy sheet structure and the kinetic of spheroidization; Xi'an: Northwestern Polytechnical University,2007.

[16] Sha Aixue, Li Xingwu, Huang Lijun, Wang Qingru. Process Analysis of BT16 Titanium Alloy Wire. Rare metal materials and Engineeri 